\title{
MedienPädagogik
}

Zeitschrift für Theorie und Praxis der Medienbildung

\section{Jahrbuch Medienpädagogik 5. Evaluation und Analyse}

Zweitveröffentlichung aus: Jahrbuch Medienpädagogik 5. (2005) Wiesbaden: VS Verlag für Sozialwissenschaften. Hrsg. v. Ben Bachmair, Peter Diepold und Claudia de Witt.

\section{Themenfelder der Medienpädagogik}

\author{
Ben Bachmair
}

Schnell ist das Ziel der Medienpädagogik mit „Medienkompetenz“ benannt. Wenn man die Geschichte der Medienpädagogik und Mediendidaktik durchgeht, dann ist mit dieser Zieldefinition schon eine Menge erreicht. Es ist ein Erfolg der Medienpädagogik als Disziplin, dass sich eine von Politik, Wirtschaft und Schule akzeptierte Zielvorstellung heraus kristallisiert hat. Reicht diese allgemeine Akzeptanz jedoch aus, um Medien als Bildungsaufgabe zu bestimmen? Will man sich der Bildungsaufgabe theoretisch wie praktisch stellen, dann geht es um nicht weniger als die Frage, wie Medien in das Verhältnis der Menschen zu sich, zu anderen, zur Kultur, zu den Dingen und Ereignissen vermittelnd eingreifen, vielleicht sogar Prägekraft entwickeln. Das Verhältnis der Menschen zu sich, zur sozialen, dinglichen und kulturellen Umwelt war und ist immer in Bewegung. In diesem Verhältnis entstanden und entstehen typische Formen von Subjektivität, verläuft Sozialisation. Subjektivität konstituiert sich immer in einem unstetigen Feld von subjektivem Innen und objektivem Außen, von individueller Erfahrung und institutioneller Festlegung, von vorgegebener Kultur und einmaliger Aneignung. Im Moment erleben wir dieses Feld als besonders unstetig. Deshalb die Frage, was sich aktuell verändert, um die für die Konstitution von Subjektivität, anders formuliert, um die für Bildung und Sozialisation maßgebliche Konstellation im Medienbereich zu entdecken. Vielleicht lassen sich so Themenfelder der Medienpädagogik neu akzentuieren. Deshalb einführend einige Stichworte zur kulturellen und gesellschaftlichen Entwicklung.

Was war prägend in den letzten Jahren?

Auffällig waren der Boom des Internet und die explosionsartige elektronische Vernetzung der Medien, die zu einem der Motoren der Globalisierung wurden. Dazu gehört jedoch auch die Implosion des dot.com-Marktes oder der Konkurs eines Mediengroßunternehmen wie der der die Kirch-Gruppe. An diesen Zusammenbrüchen zeigt sich die Krisenhaftigkeit unserer gesellschaftlichen, ökonomischen, aber auch kulturellen Entwicklung. Der 11. September 2001 hat dies in einem Ereignis und in einem Bild verschmolzen. Merkwürdig ist jedoch, wie in unseren alltäglichen Routinen dieses Bild des zentralen Turms Manhattans, den ein Flugzeug mit Feuer durchdringt, zu verblassen beginnt. Dies Bild 
überlagert sich zudem mit den aktuelleren Medienbildern, wie denen vom 2. Golfkrieg, die auch schon wieder verschwinden. Sie verblassen nicht so sehr in der ständigen Wiederkehr globaler Bilder. Es ist vielmehr die Routine des Alltagslebens, die unsere ganze Energie auf sich zieht, wobei sich der öffentliche Wahrnehmungsfokus auf die Staatsfinanzen, die Finanzierung von Renten und Krankenversicherung, zudem auf die Arbeitslosigkeit richtet. Im Moment müssen wir - oder stellvertretend Staat und personalisierte Politik - enorm viel Energie einsetzen, um diese Routine aufrecht zu erhalten. Das ist Folge der Dynamik unserer gesellschaftlichen Übergangssituation, die mit einer Enttraditionalisierung des routinierten Lebens in der Industriegesellschaft einhergeht. Im Vordergrund stehen die staatlichen Garantien, die wichtigen Bündel an Gefährdungen wie Krankheit oder Arbeitslosigkeit, die das Leben mit sich bringt, kollektiv zu versichern.

Wie kommt man argumentativ von dieser Liste ausgewählter Probleme zu medienpädagogischen Themenfeldern? Dazu empfiehlt sich nach den sich abzeichnenden allgemeinen Leitlinien zu fragen, innerhalb derer sich unsere Gesellschaft und unsere Kultur entwickeln.

\section{Enttraditionalisierung in den westlichen Industriegesellschaften}

Wir sind mitten in einem Schub der Enttraditionalisierung, der uns aus der Sicherheit der Industriegesellschaft in eine Gesellschaft individualisierter Risiken treibt. Es ist ein Schub der Enttraditionalisierung, den Theodor Adorno und Max Horkheimer als Ausgangspunkt für die Entwicklung der „Kulturindustrie“ Anfang der vierziger Jahre des vergangenen Jahrhunderts in den USA beschrieben haben. Damals ging es immer noch um den Schock der Industrialisierung, welche die Menschen aus ihren vertrauten und zumeist religiösen Interpretationsmustern und bäuerlichen Handlungsmuster drängte.

„Die soziologische Meinung, dass der Verlust des Halts in der objektiven Religion, die Auflösung der letzten vorkapitalistischen Residuen, die technische und soziale Differenzierung und das Spezialistentum in kulturelles Chaos übergegangen sei, wird alltäglich Lügen gestraft. Kultur heute schlägt alles mit Ähnlichkeit. Film, Radio, Magazine machen ein System aus. Jede Sparte ist einstimmig in sich und alle zusammen“ (Horkheimer/Adorno 1969).

Der aktuelle Schub der Enttraditionalisierung läuft in den westlichen Industriegesellschaften als Reduktion staatlicher Aufgaben, die mit einer Individualisierung der Lebensrisiken der Menschen einhergeht. Unter anderem zwingen Reduktion des Staates und Individualisierung die Menschen, ihre eigene Lebenswelt aufzubauen. Sie tun dies, indem sie ihre eigenen Interpretations- und Handlungsmuster entwickeln. Einer der aktuellen Theoretiker dieser Entwicklung, Ulrich Beck, beschreibt diesen Trend folgendermaßen: 


\begin{abstract}
„Doch bei allen Ähnlichkeiten zu diesen Themenstellungen des auslaufenden 19. Jahrhunderts werden die Menschen heute nicht aus ständisch-religiösen Gewissheiten in die Welt der Industriegesellschaft entlassen, sondern aus den Sicherheiten der Industriegesellschaft in die Turbulenzen der Weltkrisengesellschaft. Ihnen wird also das Leben nicht zuletzt mit den unterschiedlichsten, einander widersprechenden globalen und persönlichen Risiken zugemutet.

Die Industriegesellschaft setzt Ressourcen von Natur und Kultur voraus, auf deren Existenz sie aufbaut, deren Bestände aber im Zuge einer sich durchsetzenden Modernisierung aufgebraucht werden. Dies trifft auch auf kulturelle Lebensformen (z.B. Kleinfamilie und Geschlechtsordnung) und soziale Arbeitsvermögen zu (z.B. Hausfrauenarbeit, die zwar nicht als Arbeit anerkannt war, gleichwohl aber die Erwerbsarbeit des Mannes erst ermöglicht hat).

Dieser Verbrauch der kollektiven oder gruppenspezifischen Sinnreservoire (z.B. Glauben, Klassenbewusstsein) der traditionalen Kultur (die mit ihren Lebensstilen und Sicherheitsvorstellungen noch bis weit in das 20. Jahrhundert hinein auch die westlichen Demokratien und Wirtschaftsgesellschaften gestützt hat) führt dazu, das alle Definitionsleistungen den Individuen zugemutet werden.

Chancen, Gefahren, Ambivalenzen der Biographie, die früher im Familienverband, in der dörflichen Gemeinschaft, im Rückgriff auf ständische Regeln oder soziale Klassen bewältigt werden mochten, müssen nun von den einzelnen selbst wahrgenommen, interpretiert und bearbeitet werden. Chancen und Lasten der Situationsdefinition und -bewältigung verlagern sich damit auf die Individuen, ohne dass diese aufgrund der hohen Komplexität der gesellschaftlichen Zusammenhänge noch in der Lage sind, die damit unvermeidlichen Entscheidungen fundiert, in Abwägung von Interessen, Moral und Folgen verantwortlich treffen zu können“ (Beck 1993).
\end{abstract}

\title{
Der Trend zu „globalen Netzwerken der Instrumentalität“ - ein Konzept von Manuel Castells
}

Massenkommunikation ist zweifellos eine unserer kulturelle Ressourcen, die individuelle Sinnwelten mit prägt, indem sich die Menschen der Industrie- und Konsumgesellschaft auch mit den Programmangeboten und innerhalb der Medienform ihre persönlich bedeutsame Lebenswelt aufbauen. Anders formuliert heißt das, die Programmangebote der Massenkommunikation bekommen die Funktion von Bausteinen für eine persönliche Lebenswelt. Mit dieser Funktion von Massenkommunikation als kulturelle Sinnressource bzw. als Lebensweltbaustein ändern sich auch Inhalt, Form und Gefüge der Massenkommunikation. So verschiebt sich im Moment das vertraute Verhältnis von Publikum und Produzenten. Deutliches Beispiel sind dafür Casting Shows und Real Live Shows wie „Pop Stars“ oder „Big Brother“, in denen Menschen über ein Casting-Verfahren aus dem Publikum auf die Medienbühne steigen und nach einer kurzen Prominenz-Phase wieder ins Publikum und ihren Alltag zurückkehren. Also 
auch Massenkommunikation unterliegt dem kulturellen Wandel und trägt zur gesellschaftlichen Unsicherheit bei. Die kulturellen Ressource Massenkommunikation ist nicht nur Instrument der aktuellen kulturellen Veränderungen, sondern verändert sich selber dramatisch in ihrer Struktur. Dabei ist spielt auch das Internet eine wichtige Rolle, indem es nicht nur neue Präsentationsform liefert, sondern das Mediengefüge verändert. Manuel Castells beschreibt diese strukturelle Veränderung als „globale Netzwerke der Instrumentalität“. Spontan stellt man sich hierzu das Internet vor. Auch wenn das Internet sich weder so schnell noch so nachhaltig im Alltag und als Bestandteil von Massenkommunikation etablierte, wie es Ende der neunziger Jahre den Anschein hatte, so zeigt sich am Internet doch, was sich mit der Vernetzungs- und Verfügungslogik, die dem Internet zu eigen ist, verändern kann. Manuel Castells (2001) skizziert mit seiner Analyse des „Informationszeitalters“ und der als ein instrumentelles Netzwerk organisierten Gesellschaft die Umrisse, wie sich unsere Gesellschaft mit welcher Dynamik entwickelt. Er spricht von einer „informationeilen Entwicklungsweise“ (2001, S. 17), die anders als die „,agrarische“ oder die „,industrielle“ Entwicklungsweise auf das gesellschaftliche Verhältnis der Menschen zur Natur (das ist die „Produktion“), zu sich selber (das sind „Erfahrung“ und „kulturelle Identität“) und zu anderen (das ist unter anderem „Macht“) einwirkt.

Einen Aspekt dieser „informationellen Entwicklungsweise“ beschreibt Castells mit dem Hinweis auf „globale Netzwerke der Instrumentalität“, die eine „unüberschaubare Anzahl virtueller Gemeinschaften“ hervorbringt (ebd., S. 23). Dabei konstituiert sich auch eine Art von Subjektivität, die sich im Moment als „zunehmende Distanz zwischen Globalisierung und Identität, zwischen dem Netz und dem Ich“ beschreiben lässt (ebd., S. 24). In dieser Spannung bekommt „Multimedia“ die Funktion einer „symbolischen Umwelt“ (ebd., S. 415). Multimedia braucht soziale Formen, die mit Lifestyle-Szenen und Lifestyle-Milieus auch im Entstehen sind. Hierbei handelt es sich um alltagsästhetisch homogene soziale Gruppierungen, in die sich die Menschen mit Handlungsmustern des Konsumierens einwählen. Sie werden die wählbaren und fließenden Sozialformen der Postmoderne, welche die vertraute Massenkommunikation mit der industriellen Produktion und Verteilung kohärenter Medien ablösen wird.

\section{Interpretationsweisen und Diskurse ohne kulturelle Stabilität}

An dieser Stelle tritt der Konflikt zwischen der Industrie-Kultur westlicher Prägung und Kulturen mit einer anderen historischen Dynamik, wie die der muslimisch ausgerichteten Gesellschaften, zu Tage. In diesen Gesellschaften potenzieren sich die Konflikte der Enttraditionalisierung an der Grenzlinie von religiösen Lebensformen zu säkularen Lebensformen der Industriegesellschaft mit dem Primat der Zweckrationalität und Verfügbarkeit in Produktion und Kon- 
sum. Denn gleichzeitig lösen sich die mit der Industriegesellschaft verbundenen Sicherheiten auf, insbesondere die Sicherheit des demokratischen Rechts- und Fürsorgestaates. Zugleich wird das Wohlstandsversprechen des Konsums leer.

Was heißt das konkret? Es verbindet sich die Risikovariante der Arbeitslosigkeit der westlichen Industriegesellschaft mit den Deutungsrisiken postmoderner Lebensformen. Wer weiß denn, was den „Werten“ der Industriegesellschaft in der „Netzwerkgesellschaft“ mit reduziertem Staat entspricht? Ich vermute, „Werte“ entstehen als neue Varianten medienbezogener Diskurse, die jedoch innerhalb der Grenzen sozialer Milieus stattfinden. Was bringt dieser Diskurs jedoch denjenigen, die keinen Job finden? Auch diese Diskurse stehen innerhalb fragmentierter Bezugsrahmen, denen die Sicherheit der mit generellem Gültigkeitsanspruch ausgestatteten Werte fehlt.

Gleichzeitig stellt sich die Frage nach Macht und Herrschaft in den westlichen Industriegesellschaften breiter als nur die nach der Kontrolle der Exekutive. Diese Frage erscheint im Moment meist nur vage im Boulevard-Stil, wer denn den reduzierten Staat besitzen oder wer den Staat kommandieren wird. Dies ist jedoch alles andere als eine vordergründige Macht-Frage für die staatlichen und kulturellen Newcomer in der Industriegesellschaft. Sie ist unmittelbar verbunden mit der Strategie, wie die Deutung dieser Welt geschieht, wie Deutung, mediale Angebote und die Lebensformen der individualisierten Risiken aufeinander bezogen sind. Der Sender Al Jazeera ist dafür kein schlechtes Beispiel.

In dieser kulturellen Frage und deren Beantwortung in der Form medialer Angebote und Lifestyle-Arrangements steckt enorm viel gesellschaftliches Gewaltpotenzial. Somit werden kulturelle Fragen der Deutung zu politischen und auch zu moralischen Themen. Die an das Selbstverständnis eines Mitglieds einer Kultur gebundene Diskurse und Deutungen (umgangssprachlich formuliert geht es um „Identität“) werden damit genuin politisch.

Was gemeint ist, lässt sich am Kopftuch der deutschen Muslimin sehen, die Lehrerin in der staatlichen, öffentlichen Schule werden will, die sich zugleich schämt, mit unbedecktem Haar vor eine Schulklasse zu treten. Dieser Fall lag beim Verfassungsgericht der Bundesrepublik zur Entscheidung, wobei es um etwas anderes ging als um Fragen der Selbstverwirklichung einer jungen Frau. „Selbstverwirklichung“ war die politische Frage Ende der sechziger Jahre, in der es um die Definition subjektiv bestimmter Lebensformen innerhalb der Industriegesellschaft ging. Das damalige politische Thema war die Freiheit in der Industriegesellschaft, die kulturell mit dem Wunsch nach Selbstverwirklichung und mit der freien Wahl der Lebensformen beantwortet wurde. Jetzt geht es um etwas politisch Neues, das als Frage der Identität in die Neudefinition an der Grenze von Religiös-Vorindustriell und Postindustriell eingeht. Vermutlich markiert die Frage nach Identität und Kopftuch die gleiche Entwicklungslinie wie die Bilder des Flugzeugs im Turm des World Trade Centre. 
Alltag und Massenkommunikation als die sich verändernden Domänen der Industriegesellschaft

Es gibt einen zentralen Unterschied: Die Kopftuch-Frage bleibt innerhalb des Alltagslebens, die der 11. September und seine Kriegsfolgen definitiv verlassen und auch zu zerstören versucht hat. Im Alltagsleben als der zentralen Domäne der Industriegesellschaft hat sich die Massenkommunikation etabliert. In der Entwicklungslinie des Alltags verändert sich auch die Massenkommunikation und umgekehrt: Mit der Veränderung der Massenkommunikation korreliert das Alltagsleben.

Die aktuelle Umgestaltung der Massenkommunikation läuft auch auf eine neue Mensch-Medienbeziehung hinaus, bei der sich Medien in komplexe Arrangements mischen und in denen Medien die Funktion von „Providern“ symbolischer Angebote bekommen. Auch in den westlichen Industriegesellschaften bringen Angebote wie Big Brother viel Unklarheit, worum es sich denn dabei eigentlich handelt. Bei Big Brother gab es den öffentlichen Diskurs, was das Thema dieses Genres sei: Zurschaustellung naiver Alltagsmenschen, Zerstörung der Persönlichkeitsrechte von Menschen, Spaß an den Handlungsmustern von Leuten wie du und ich?

Die offenen Fragen zur Veränderung der Massenkommunikation reichen von den traditionellen Themen wie Gewaltdarstellungen (z.B. Mangas) und Pornografie (z.B. Internet) bis zu Tauschbeziehungen bei immer währenden Internet-Auktionen oder Telefonnummer vom Typ 0190-, die Kommunikation, Rat oder verbalen Sex gegen Geld versprechen.

\section{Kulturelle Übersetzung}

Für die politische Dimension dieser Fragen hat der Soziologie und Philosoph Jürgen Habermas einen bemerkenswerten Vorschlag formuliert. Auch Habermas ist sich sicher, dass der aktuelle gesellschaftliche Umbruch als Konflikt dem Konflikt des Übergangs von der religiös geprägten Ständegesellschaft zur säkularen Industriegesellschaft entspricht. Als gelungen sieht er den damaligen Übergang der Deutungsmuster an, den es heute, bezogen auf den Konflikt mit den Deutungsmustern der muslimischen und der westlichen Industriegesellschaft, ebenfalls zu meistern gilt. Er sieht als aktuelle historische Aufgabe die Übersetzungsarbeit, eine kulturelle Übersetzungsarbeit, die an den historischen Bruchlinien von Mythos und kirchlich verfasster Religion ebenso gelungen ist, wie an der Bruchlinie der Säkularisierung der Industriegesellschaft: ${ }^{1}$

1 Auszug aus dem Text der Rede von Jürgen Habermas in der Paulskirche vom Oktober 2001. Frankfurter Rundschau vom 15.10.2001: „Der Riss der Sprachlosigkeit. Nach den Terrorakten: Der Friedenspreisträger des deutschen Buchhandels, Jürgen Habermas, über die Bedeutung der Säkularisierung in modernen Gesellschaften. Die Attentate vom 11. September in den USA haben auch in der Rede von Jürgen Habermas in der Frankfurter Paulskirche ihre Spuren hinterlassen. In den Terrorakten sieht der 72-jährige Starnberger Philosoph die Spannung zwischen säkularer Gesellschaft und Religion in besonderer Weise explodiert.“ 


\begin{abstract}
„Die postsäkulare Gesellschaft setzt die Arbeit, die die Religion am Mythos vollbracht hat, an der Religion selbst fort. Freilich nicht in der hybriden Absicht einer feindlichen Übernahme, sondern aus dem Interesse, im eigenen Haus der schleichenden Entropie der knappen Ressource Sinn entgegenzuwirken. Der demokratisch aufgeklärte Commonsense muss auch die mediale Vergleichgültigung und plappernde Trivialisierung aller Gewichtsunterschiede fürchten. Moralische Empfindungen, die bisher nur in religiöser Sprache einen hinreichend differenzierten Ausdruck besitzen, können allgemeine Resonanz finden, sobald sich für ein fast schon Vergessenes, aber implizit Vermisstes eine rettende Formulierung einstellt. Eine Säkularisierung, die nicht vernichtet, vollzieht sich im Modus der Übersetzung. Das ist es, was der Westen als die weltweit säkularisierende Macht aus seiner eigenen Geschichte lernen kann.“
\end{abstract}

Diese Übersetzungsarbeit zwischen Kulturen ist genauso notwendig wie die Übersetzungsarbeit zwischen den Generationen und zwischen den neuen Sozialformen der Postmoderne wie Fan-Gruppen, Milieus oder Szenen.

\title{
Herrschaft und Legitimation
}

Es gibt offensichtliche Machtzusammenhänge im Mediengeschäft. Dafür stehen Namen wie Murdoch, Berlusconi oder der des mittlerweile gescheiterten Kirch. Wichtig ist auch die Frage, wer sich welchen Medien-Zugang leisten kann. Das ist die Machtfrage in der Konsumgesellschaft von Seiten der Nutzer. Die Verschuldung junger Leute über ihre Handys ist hier ein Punkt, der nachdenklich macht. Die Kosten für die Nutzung von Internet-Studienplattform, die sich Studierenden nicht leisten können, zeigt die künftige Relevanz für Bildung. Dabei sollte die traditionelle Frage einer Demokratie nicht vergessen werden, dass die Herrschaft des Volkes nur in Zusammenhang mit Bildung nicht zur Stammtischideologie verkommt.

Es gibt weniger leicht zu durchschauende Machtkonstellationen, die auch der Legitimation bedürfen. Dazu gehören neue Formen „symbolischer Texte“, die sich die Menschen aus den Gütern, Medien und Konsumgewohnheiten zu Lifestyle-Einheiten zusammenmischen.

\section{Mit Lifestyle-Arrangements begrenzen oder öffnen}

Wie sich mit den textartigen Geweben der Lifestyles auch Formen von Macht und Herrschaft verbinden, haben vor geraumer Zeit schon Pierre Bourdieu mit seiner Analyse kultureller Ressourcen und Stuart Hall mit der Analyse der Organisation von Massenkommunikation skizziert. So steht für Bourdieu „die Art der Beziehung, die jedes einzelne Individuum mit der gesamten Sphäre der kulturellen Produkte verbindet“ „in engstem Konnex mit seiner Stellung in diesem Felde“ (1991, S. 107). Konkret geht es um die „feinen Unterschiede“ (Bourdieu 1989) die mit der Verwendung kultureller Produkte einhergehen. Diese 
„feinen Unterschiede“ reichen von den Kleidern, die sich die Menschen auswählen, über die dazu passenden Autos bis zu den Fernsehgenres. Die Felder und Produkte der „feinen Unterschiede“ wachsen mittlerweile zu Lifestyles zusammen. Mit der Mischung einzelner Medien in die komplexen Arrangements individueller Lebenswelten bleibt die gesellschaftliche wie die individuelle Auseinandersetzung um Legitimität bzw. um entsprechende Ansprüche unvermeidlich. Gerhart Schulze (1992) hat gezeigt, wie Menschen sich in und mit alltagsästhetisch konstituierten Milieus und Handlungsschemata grundlegend in der Welt verankern. Weil eine alltagsästhetisch verfasste Welt wählbar ist, steht jede einzelne in Konkurrenz zu anderen Lifestyle-Welten. Deren Legitimität ist mit ihren jeweiligen leitenden Repräsentationsformen verbunden. Diese leitenden Repräsentationsformen können Medien-Genres wie Popstars oder Big Brother sein, aber auch Wohnungseinrichtungen, Freizeitvorlieben usw.

Dabei kommt die von Stuart Hall formulierte Dynamik des Herstellens und Rezipierens von Medien, Hall beschreibt sie als „Encoding“ und „Decoding“ (Hall 1980), ihr politisches Gewicht. In der Auseinandersetzung über Deutungsmacht, Autonomieerfahrungen, Widerstandpotentiale oder Unterwerfungsprozeduren bei der Aneignung von Themen oder Darstellungsweisen definieren sich Macht- und Herrschaftsverhältnisse in ihrer kulturellen Erscheinungsform (vgl. Dörner 1999).

\section{Erkan liest, jedoch „nur“ vom Fernsehbildschirm}

Diese Macht-Frage in Sachen Medien stellt sich heute gerade auch an der Konfliktlinie zwischen kulturellen Traditionen. Das folgende Beispiel zur Lesekompetenz eines 11-jährigen türkischen Jungen beleuchtet gleichermaßen die Konfliktlinie zwischen Muslimen und Europäern, wie die Konfliktlinie zwischen gesellschaftlichen Schichten von Arbeitern und schulbezogener Mittelschicht.

Erkan ist 11 Jahre alt und hat einen türkischen Pass. Er ist in der Bundesrepublik geboren und aufgewachsen. Seine Schulkarriere ist in zwei Bundesländern mit recht unterschiedlichen Lehrplänen weitgehend gescheitert. Seine Eltern sprechen sehr gut deutsch, haben sich voll auf Deutschland eingelassen, unterstützen auch alle schulbezogenen Aktivitäten ihrer Kinder, z.B. indem sie sich ihre Wohnung in der Gegend der Schule gesucht haben. Jedoch eine Art von Bildungsbündnis mit der Schule zu knüpfen, ist nicht gelungen. So wollen Erkans Lehrer ihn zwangsweise in die Sonderschule einweisen, weil er in der Regelschule nicht mehr mitkommt.

Worin liegt Erkans Schulproblem begründet? Vermutlich ist Erkan nie in das deutsche Bildungssystem ,eingestiegen“. Er lässt auch keine Alternative zur Idee aufkommen, etwas anderes als sein Großvater sein zu wollen, nämlich ein Türke. Was wundert es, dass er kein deutsches Wort mit einem redet, auch wenn man bei ihm zu Hause im Wohnzimmer sitzt. Statt dessen konzentriert er sich auf den Fernseher. Es läuft „Formel 1“, das Autorennen auf RTL. „Formel 1“ ist eine der von Jungen dieses Alters favorisierten TV-Sendungen. Als Erkans Papa von der Arbeit nach Hause kommt, fasst Erkan in Windeseile die Ergeb- 
nisse von „Formel 1“ zusammen. Papa ist sehr interessiert, denn für Autos wendet er generell viel Zeit auf, kann sie selber reparieren und spricht ebenso gern wie kompetent darüber. Erkan hat bei „Formel 1“ offensichtlich genau zugesehen und sich die Ergebnislisten gemerkt, die er für seinen Vater versprachlicht und interpretiert, selbstverständlich auf Deutsch. Voraussetzung dazu ist die Fähigkeit, Listen innerhalb von Sportsendungen wie „Formel 1“ zu lesen. Solche Listen sind sogenannte diskontinuierliche Texte, die nach den OECD-Kriterien zur modernen Lesekompetenz gehören. Für die Schule zählt jedoch Lesekompetenz in der Regel nur, wenn sie zum Lesen gedruckter Texte verwendet wird. Die Repräsentationsform des diskontinuierlichen Lesetextes auf dem Fernsehbildschirm ist dagegen schulisch ohne Wert.

Schon mit unterschiedlichen Repräsentionsformen traditioneller Texte verbinden sich gesellschaftliche Maßstäbe, die kaum bewusst sind und doch über schulischen und beruflichen Erfolg mitentscheiden. Wie gravierend sind erst die über Jahrhunderte entstandenen Bewertungen von Bildern im Kontext von Religion oder von Unterhaltung! Wenig vorstellbar ist eine Koexistenz zwischen den neuen alltagsästhetisch organisierten Lifestyle-Texten inklusiv ihrer inhärenten Lebenskonzepte einerseits und den Wertmaßstäben an der Bruchlinie vorindustrieller Clans zu „globalen Netzwerken der Instrumentalität" andererseits.

Auf der Suche nach gangbaren Wegen - die Frage nach Medienethik und Bildung Jürgen Habermas hat mit dem Blick des Soziologen und Philosophen die Aufgabe der kulturellen Übersetzungsarbeit entdeckt, indem er die kulturellen Brüche zwischen Mythos und Religion, zwischen agrarischer und industriell geprägter Gesellschaft bedacht hat. Kulturelle Übersetzungsarbeit haben beispielsweise die institutionalisierten Kirchen geleistet, sicherlich nicht uneigennützig, indem sie Mythen in ihre eigene Weltdeutung und Sinngebung integrierten. Die Industriegesellschaft hat sich dazu beispielsweise des Museums oder des Zoos bedient, auch der nicht-positivistischen Wissenschaft oder des Geschichtsunterrichts ebenso wie der Literatur und des Films. Welche kulturelle Übersetzungsarbeit gelingt in den „globalen Netzwerken der Instrumentalität“ mit den institutionalisierten Medien wie Fernsehen oder der neuen Massenkommunikation in den Lifestyle-Formen? So liefert die Integration des geschriebenen Textes in der Sportberichterstattung des Fernsehens Erkan die Möglichkeit, in seinem Alltagsleben den geschriebenen Text als sinnvoll zu erleben. Lifestyle-Formen tragen in sich die Chance, auch das Kopftuch der deutsch-muslimischen Lehrerin kulturell zu übersetzen.

Darüber hinaus sind andere Wege begehbar zu machen, beispielsweise auch mit pädagogischen Schlüsselideen wie die des Jean-Jacques Rousseau von der Natur des Menschen. Eine Neuinterpretation der grundlegenden Idee von Jean- 
Jacques Rousseau von der Natur des Menschen könnte den Ausgangspunkt einer anthropologischen, vom Wesen des Menschen ausgehenden Definition von Subjektivität und Bildung bieten. In diesem Kontext scheint es mir notwendig, zwei Ideen in Bezug auf Medien sowie auf die alte und auf die neue Form der Massenkommunikation genauer zu untersuchen.

Es ist erstens der Gedanke der Verletzlichkeit der Menschen, woraus sich die Aufforderung zur empathischen Parteinahme für die Unerfahrenen ergibt. Empathie ist zusammen mit Realitätsbezug und Ich-Stärke eine der zentralen Triebkräfte der Sozialisation. Es ist zweitens eine Bildungsidee, die sich aus den neuen und Bedeutung schaffenden Formen der Medienrezeption der Massenkommunikation ergibt. Es geht um den „Eigensinn der Menschen, der mit dem Gedanken vom „Eigensinn der Welt zu verknüpfen ist. „Eigensinn“ verweist sowohl auf die für heutige Mediennutzung prägende Bedeutungskonstitution als auch auf die Unverfügbarkeit von Menschen und Sachverhalten in einer konsumorientierten Lebensform.

Vom Eigensinn der Kinder und Sachen bis zum Anker in einer fließenden Welt der Zeichen Im Gemenge der Medien und Ereignisse sowie in der Sicherheit aber auch in der individuell riskanten, alläglichen Lebensgestaltung entwickelt sich die pädagogische Frage- und Aufgabenstellung. Dabei geht es im ersten Schritt um Handlungs- und Aneignungsmuster, also nicht mehr vorrangig um die Medienerlebnisse oder gar um die Medien selber.

Die neue und eher konsumierende, mediengerechte Form der Aneignung hat eine postmoderne Dynamik, die nichts mehr mit Pauken zu tun hat. Kinder eignen sich die Welt in einer spezifischen Erlebnisweise an. Diese Erlebnisweise hat sich mit Konsumorientierung und Individualisierung entwickelt, bei der Medien, insbesondere Fernsehen, einen nicht zu unterschätzenden Anteil beitrugen. Die heutige Kindergeneration ist im Trend zur Individualisierung und mit der Notwendigkeit aufgewachsen, individuelle Lebenswelten gerade auch mit den symbolischen Materialien der Medien aufzubauen. Damit beginnen Kinder und Jugendlichen ihre Erlebnisweisen zu verändern. So stehen sie tendenziell im Mittelpunkt einer eigenen, subjektiven Welt, die sich anders aufbaut als die Lern- und Sachwelt der Schule. Diese postmoderne Welt entsteht um das eigene Ego in Prozessen der Bedeutungskonstitution. Bedeutungskonstitution ist das Gegenmodell zur passiven Medienrezeption. Kinder eignen sich das symbolische Material von Konsumobjekten, Medien und Ereignissen individuell an, indem sie ihnen perspektivisch und innerhalb von Relevanzrahmen subjektive Bedeutung verleihen. Die jeweilige Medienwelt ist einer dieser Relevanzrahmen.

Sich vergewissern: Reflexivität für Bastler in Kulturinszenierungen

Medienkompetenz ist sicher eine Form, wie Kinder eine reflexive Beziehung zu Medien als Teil von Kulturinszenierungen entwickeln. Im Mittelpunkt sollte dabei ein KompetenzBegriff stehen, der sich an den der Textkompetenz von 
Pisa anlehnt: „Informationen ermitteln“, „Textbezogenes Interpretieren“, „Reflektieren und Bewerten“. Daneben gibt es eine Fülle von Möglichkeiten, Kindern emotionale, soziale und rationale Distanz und Auseinandersetzung anbieten. Auf einer weiteren Stufe von Reflexivität sind auf Medien und Konsum bezogene Lebenswelten zu ordnen und zu gestalten.

\section{Eigensinn von Kindern, Dingen und Ereignissen}

Kinder sollen nicht zu Objekten von Zwecktexten oder Einschaltquotenkalkül werden. Ihr Eigensinn in der Doppelbedeutung sowohl widerständig zu sein als auch standardisierten Medienangeboten eigenständig Sinn zu geben ist deshalb eine wichtige Bewertungsdimension für Fernsehangebote. Dies ist auch eine Komponente von Medienschutz. Wichtig ist, ob und wie Sender, Warenproduzenten, Entertainment-Unternehmen aber auch Schule die Kinder als Subjekte adressieren, welches implizite Kinder- und Konsumentenbild sie unterstellen. Gleichzeitig existiert auch in der erlebnisorientierten Gesellschaft die Welt der Sachverhalte und Ereignisse als etwas Eigenständiges mit eigenem Wert und Sinn, das über das individuelle Verfügen hinaus reicht. Es geht in dieser Bewertungsdimension also um die Funktion von Medien für die Realitätserklärung, deren konsequente Form das Lehren und Lernen ist.

\section{Kontexte herstellen in der Welt als Supermarkt}

Die Aufgabe, sich in einer vielschichtigen und auch brüchigen Welt die eigene Lebenswelt aufzubauen, ist nicht nur für Kinder eine kräftezehrende und riskante Aufgabe, zu deren Bewältigung sie Hilfe brauchen. Nicht erst mit der Globalisierung liefert Fernsehen bzw. andere elektronische Medien eine Fülle von Formaten und Genres, die sich nicht allein auf der Basis kultureller Tradition erklären. Gängig ist in diesem Zusammenhang die Vorstellung, Kinder sollten beim Fernsehen natürlich nicht allein gelassen werden; zuhörende, erklärende oder auch beruhigende Eltern bzw. Bezugspersonen sollten sie dabei begleiten. Dies nicht allein der Familie zu überlassen, ist auch Aufgabe der Medienanbieter. Es gilt insbesondere, wenn Medienprogramme den angestammten Kulturkontext verlassen und Kinder sie ohne tradierten oder vertrauten Bezugsrahmen, also ohne eigene Genrekompetenz, rezipieren.

Versuch, einen Themenkatalog zu bestimmen

Lässt sich aus einer Analyse der aktuellen gesellschaftlichen und kulturellen Situation ein Bezugsrahmen für Themenfelder der Medienpädagogik herausarbeiten? Sicher nicht eindimensional oder deduktiv ableitend, zum Beispiel indem aus den Analysen und Erklärungsversuchen der Kulturtheorie (Ulrich Beck, Anthony Giddens oder Gerhard Schulze) verpflichtende Aufgaben oder sogar noch Normen für Medienpädagogik hergeleitet werden. Medienpädagogen leben und denken jedoch in dem Feld der Kultur, dessen Wandel theoretisch abgesteckt ist. Deswegen empfiehlt es sich, die medienpädagogischen Aufgaben 
kulturtheoretisch zu bedenken und zu fragen, wie wir als Medienpädagogen Kinder und Jugendliche unterstützen und fördern. Dabei reagieren wir auch auf die aktuelle krisenhafte Umbruchssituation. Nur zur Erinnerung im folgenden eine Liste, die deren zentralen Punkte aufführt:

- Enttraditionalisierung der westlichen Industriegesellschaft,

- Individualisierung der Lebensrisiken (Krankheit, Arbeit) und Lebensformen,

- Globalisierung als Lebensform der „globalen Netzwerke der Instrumentalität“,

- Ästhetisierung der Lebensformen und die Verschiebung der Sozialisationsperspektive von einer objektiven gesellschaftlichen Wirklichkeit zur individuellen „Erlebnisrationalität“.

Auf dieser aktuellen gesellschaftlichen und kulturellen Problemfolie gestalten sich die Felder der Mensch-Medien-Beziehung spezifisch, vielleicht auch neu. Diese Felder werden u.a. von den folgenden Kräften beeinflusst, die eine medienpädagogische Antwort erforderlich machen.

\section{Macht und Herrschaft}

- Eine Demokratie braucht Kontrolle der Medien durch Politik und Publikum. Kritische Medien- und Institutionsanalyse sowie deren Einübung in Formen wie die der Bürgerkanäle sind institutionell zu stabilisieren und weiterzuentwickeln .

- Die in Medienformate und Darstellungsformen (Buch/Bildschirm) verwobenen „feinen Unterschiede“ (Pierre Bourdieu) in der kulturellen und gesellschaftlichen Hierarchie sind bewusst zu machen.

- Zu fragen ist, welche medialen Kulturtechniken und welche Formen von Medienkompetenz notwendig sind, um in den „globalen Netzwerken der Instrumentalität“ sozial, kulturell und wirtschaftlich zu bestehen? Welche federn die individualisierten Risken ab? Welche unterstützen die Teilhabe am politischen und kulturellen Leben?

- Der Zugang zu Bildungsangeboten im Internet (z.B. Studienplattformen) darf weder an formalen Bildungsvoraussetzungen noch an finanziellen Ressourcen von Familien oder Bildungseinrichtungen scheitern.

\section{Interpretationsweisen und Diskurse}

- Eine Demokratie braucht ein kritisches Medienpublikum, das sich traditioneller, aber auch neuer Medienformate beim politischen und gesellschaftlichen Diskurs bedient. (Die Nachricht braucht Weiterentwicklung und Ergänzung, auch durch LifestyleFormate.)

- Institutionalisierte Bildung (Schule) und Unterhaltung sowie deren jeweils typische Textkompetenz und Formen der Welterklärung sollen miteinander 
versöhnt werden, so wie es das Modell des öffentlich-rechtlichen Rundfunks schon lange praktiziert. Das Konzept der Offenen Schule liefert dazu didaktische Ansatzpunkte. Hilfreich sind auch die Vorstellungen von PISA zu Funktion von Texte und von Textkompetenz. (PISA und deren Teiluntersuchung zum Verhältnis von Lesekompetenz und Freizeit zeigen aber auch, wie naiv die institutionalisierte Pädagogik gegenüber der Mediennutzung von Jugendlichen ist.)

- Zur Offenen Schule gehört die Offenheit für mediale Darstellungsformen und Medienformate (Textsorten) schulferner Bevölkerungsgruppen.

- Die von Jürgen Habermas vorgeschlagene „kulturelle Übersetzung“ ist auch eine Aufgabe der Medien und der Medienpädagogik.

- Kulturelle Übersetzung in einer fragmentierten Gesellschaft: In einer fragmentierten Gesellschaft geht es auch um die Koexistenz ethnisch oder religiös geprägte Lebensformen mit traditionell säkularen Lebensformen sowie mit neuen Lebensformen des Lifestyle. Dabei spielen jeweils spezifische, tradierte Leitmedien und deren etablierten Aneignungsformen eine Rolle, z.B. das jeweilige heilige Buch.

- Kulturelle Übersetzung zwischen Anbietern und Nutzern neuer Genres ohne vertrauten kulturellen Kontext: Es braucht die kulturelle Übersetzung zwischen den neuen Medienformaten wie Pop Stars oder Yu-Gi-Oh und den tradierten, um den Diskurs zwischen deren Nutzern zu ermöglichen. Dabei ist in besonderem Maß auf die Medienpräferenzen von Jugendlichen einzugehen. Die Globalisierung der Medienangebote (z.B. Mangas) macht diese Übersetzung zudem notwendig.

- Zwischen den Generationen und deren bevorzugten Genres und Formaten gibt es eine Konfliktlinie, die sich deutlich zwischen Lehrern und Schülern erkennen lässt. Im Generationenkonflikt in der Schule spielen auch generationsspezifische Medien- und Genrevorlieben eine Rolle.

\section{Subjektentwicklung und Erziehung}

- Eine wichtige medienpädagogische Aufgabe ergibt sich aus der Fragmentierung des Medien- und Konsumangebotes und ihres Transfers in die verschiedensten regionalen und sozialen kulturellen Kontexte. So erscheint die Welt als globaler Supermarkt, in dem auf den ersten Blick alles möglich ist, jede Wahl und Kombination Sinn macht. Kinder und Jugendlichen brauchen jedoch überschaubare Rahmen und Kontext, wobei auch die angestammte Kultur als Handlungs-, Sinn- und Bewertungsrahmen wichtig ist. Rekontextualisierung ist das Stichwort für die medienpädagogische Aufgabe. ${ }^{2}$

2 Ein Beispiel dafür ist das Begleitprogramm zur WWF-Wrestling, mit dem ein Fernsehsender sich die Aufgabe gestellt hatte, ein Fernsehprogramm aus dem amerikanischen Unterhaltungskontext für ein deutsches Kinderpublikum mit Hilfe von Trailern zu „dekonstruieren“ und als Inszenierung durchschaubar zu machen (vgl. Bachmair/Kress 1996, S. 197-218). 
- Kinder und Jugendliche brauchen Hilfe, um sich neue Medienformate ohne kulturell vertrauten Aneignungs- und Interpretationskontext selbständig zu erschließen und nutzbar zu machen. Dabei ist auf die neuen Formen der Re-Interpretation medialer Angebote, bei denen wie schon beim traditionellen Text auch, die Leser aktiv die Bedeutung eines Textes herstellen, Rücksicht zu nehmen. („Die Verstehensleistung stellt eine Konstruktionsleistung des Lesers bzw. der Leserin dar, bei der der Inhalt des Textes aktiv mit bereits vorhandenem Wissen in Beziehung gesetzt wird“; Pisa 2000, 2001, S. 78).

- Das immer währende Konsumversprechen der Industriegesellschaft macht es Kindern und Jugendlichen alles andere als leicht, sich nachdenklich, distanziert, kritisch, d.h. reflexiv zur Welt zu stellen. Sich in dieser Welt auch als Person zu vergewissern, ist eine komplizierte Aufgabe. Traditionell hat Pädagogik dies mit dem Begriff der Bildung thematisiert, der tauglich gemacht werden müsste, damit sich auch Bastler in Kulturinszenierungen reflexiv zur Welt verhalten können? Was bietet dazu Medienpädagogik?

- Medienkompetenz ist sicher eine Form, wie Kinder und Jugendliche eine reflexive Beziehung zu Medien als Teil von Kulturinszenierungen entwickeln. Im Mittelpunkt sollte dabei ein Kompetenz-Begriff stehen, der sich an den der Textkompetenz von Pisa anlehnt: „Informationen ermitteln“, „Textbezogenes Interpretieren“, „Reflektieren und Bewerten“.

- Die Enttraditionalisierung in der Herstellung und Rezeption kultureller Produkte führt bei den Medien zu einer notwendigen Eigenaktivität der Rezipienten, nämlich innerhalb der eigenen Lebenswelt und der eigenen Handlungsperspektive standardisierten Programmangeboten persönlichen Sinn abzugewinnen. Sinn herzustellen ist deshalb auch eine von der Medienpädagogik zu unterstützende Leistung von Kindern und Jugendlichen als Rezipienten. Dazu brauchen sie empathische Unterstützung, um die Medienprodukte sich eigensinnig, also auch sperrig, verfügbar zu machen. Gleichzeitig gilt es jedoch auch, den Eigenwert, den Eigensinn, also die Unverfügbarkeit der Welt erfahrbar zu machen. Konsumierbare Medien liefern eine scheinbar verfügbare Welt ins Haus. Zur medialen Bildung gehört auch die Erfahrung, dass die Welt, das sind die Dinge und Ereignisse, ihren eigenen und unverfügbaren Sinn hat, also ihren Eigensinn besitzt.

- Kinder sollen nicht zu Objekten von Zwecktexten oder Einschaltquotenkalkül werden, sollen jedoch auch den Eigenwert medialer Angebote in der Auseinandersetzung mit einem Bildungskanon medialer Angebote erfahren.

- Jugendmedienschutz: Der Jugendschutzgedanke der ungestörten Persönlichkeitsentwicklung ist gegenüber der juristischen Operationalisierung, die diesen Gedanken zweitrangig macht, deutlich zu machen und in den Wahrnehmungshorizont der Politik zu bringen. Zudem ist es notwendig, mit den Internetanbietern und ihrem Jugendschutzgremium ins verbindliche und verantwortliche Gespräch zu kommen, um sie zu einer ähnlich medienpädagogischen Sicht anzuregen, wie das für Buch, Kino und Fernsehen schon erreicht wurde. 


\section{Literatur}

Bachmair, B./Kress, G.: Begleitprogramm für eine Höllen-Inszenierung. In: Bachmair, B./Kress, G. (Hrsg.): Höllen-Inszenierung Wrestling. Beiträge zur pädagogischen Genre-Forschung. Opladen 1996, S. 197-218.

Beck, U.: Vom Verschwinden der Solidarität. Individualisierung der Gesellschaft heißt Verschärfung sozialer Ungleichheit. In: Süddeutsche Zeitung Nr. 36, Samstag/Sonntag, 14./15. Februar 1993.

Bourdieu, P: Die feinen Unterschiede. Frankfurt a.M. 1989.

Bourdieu, P: Zur Soziologie der symbolischen Formen. 4. Auflage, Frankfurt a.M. 1991 (französische Originalausgabe 1970).

Castells, M.: Das Informationszeitalter I. Die Netzwerkgesellschaft. Opladen 2001.

Dörner, A.: Medienkultur und politische Öffentlichkeit: Perspektiven und Probleme der Cultural Studies aus politikwissenschaftlicher Sicht. In: Winter, R./Hepp, A. (Hrsg.): Kultur - Medien - Macht. Cultural Studies und Medienanalyse. Opladen/Wiesbaden 1999.

Hall, St.: Encoding/Decoding. In: Hall, St./Hobson, D./Lowe, A./Willis, P. (Hrsg.): Culture, Media, Language. London 1980, S. 128-138.

Horkheimer, M./Adorno, Th. W: Kulturindustrie, Aufklärung als Massenbetrug. In: Horkheimer, MJ Adorno, Th. W: Dialektik der Aufklärung. Frankfurt 1969, S. 128-176 (Originalausgabe: New York 1944).

PISA 2000. Basiskompetenzen von Schülerinnen und Schülern im internationalen Vergleich. Opladen 2001, S. 78.

Schulze, G.: Die Erlebnisgesellschaft. Kultursoziologie der Gegenwart. Frankfurt a.M. 1992. 